\title{
Ein Fall von Angioma cavernosum des Gehirns.
}

\author{
Von \\ Dr. W. J. Sweasey Powers. \\ (Aus dem Pathologischen Institut des Königin Augusta-Hospitals zu Berlin \\ [Vorstand: Prof. Dr. Oestreich].) \\ Mit $\check{5}$ Textfiguren.
}

(Eingegangen am 26. März 1913.)

Seitdem Luschka ${ }^{1}$ ) ein Angioma cavernosum des Gehirns beschrieben hat, sind gleiche Geschwülste in geringer Zahl von $O p$ penhei ${ }^{2}$ ), Engelhart ${ }^{3}$ ), Bruns ${ }^{4}$ ), Astwazaturoff ${ }^{5}$ ), Hadlich ${ }^{6}$ ), Lorenz ${ }^{7}$ ), veröffentlicht worden. In allen diesen Fällen sind es besonders zwei Fragen, welche erörtert werden, die eine nach der Lage der Geschwulst, die andere nach dem Ausgangspunkt. Es dürfte sich empfehlen, die häufiger zu beobachtenden Angiome bzw. Varicen der Pia von den in der Gehirnsubstanz selbst entstandenen und dort gelegenen Angiomen zu trennen. Der im Folgenden beschriebene Fall gehört der letzteren Art an und zeigte der mikroskopischen Untersuchung einiges Beachtenswerte, um dessentwillen er mitgeteilt wird.

Klin is ch bot der Fall den in solchen Zuständen üblichen SymptomenKomplex der Epilepsie dar.

Frau B., 45 Jahre alt, hat 3 gesunde Kinder, wurde am 28. März 1912 auf die chirurgische Abteilung des Augusta-Hospitals (Geh. Rat Prof. Dr. F. Kra use) gebracht. Sie litt an epileptischen Krämpfen, die nach Aussage des Mannes erst etwa seit $1 / 2 \mathrm{Jahr}$ aufgetreten waren. In den letzten beiden Tagen war ein Anfall dem andern gefolgt und die Ruhepausen zwischen ihnen immer seltener geworden. Eine Ursache für die Epilepsie weiß der Ehemann der Kranken nicht anzugeben.

Bei der Aufnahme liegt sie im Status epilepticus mit klonischen Krämpfen in allen Extremitäten, cyanotisch und mit vollkommen erloschenem Sensorium. Auch in den kurzen Pausen zwischen einzelnen Anfällen ist die Kranke vollkom. men benommen. Urin und Kot läßt sie unter sich. Cornealreflex erloschen, Pupillar-

1) Cavernöse Blutgeschwülste des Gehirns. Virchows Archiv 6. 1854.

2) Monographie der Hirngeschwülste, II. Aufl., S. 222. 1902.

3) Zur Frage der Dauerheilung nach operativer Behandlung der traumatischen Jacksonschen Epilepsie. Deutsche med. Wochenschr. 1904, Nr. 3, S. 7.

4) Die Geschwülste des Nervensystems, Nr. 3, S. 7. Berlin 1908.

5) Úber die kavernösen Blutgeschwülste des Gehirns. (Zur Kasuistik der Pseudomeningitis.) Frankf. Zeitschr. f. Pathol. 8, 481, H. III.

8) Ein Fall von Tumor cavernosus des Rückenmarkes. Virchows Archiv 172.

7) Kavernöses Angiom des Rückenmarkes mit tödlicher Blutung. Inaug.-Diss. Jena $1901 / 02$. 
reflex erhalten. Die Hand wird etwas eingekrampft gehalten, der Kopf ist dauernd nach links verdreht.

Wegen der starken Pulsbeschleunigung und drohenden Herzschwäche werden Herzmittel und Hypnotica gegeben.

Da die Kranke aus dem Status epilepticus nicht herauszubringen ist, geht sie am folgenden Abend (30. März) zugrunde.

Bei der Sektion (Prof. Dr. Oestreich) erwiesen sich die Hirnhäute als unverändert, auch wurden keine Veränderungen an den Gefäßen oder auf der Hirnoberfläche gefunden. Die Gyri und Sulci waren normal geformt.

Ein Frontalschnitt durch den hinteren Teil des 2. und 3. Gyrus frontalis auf der linken Seite zeigte, in das Hirnmark eingebettet, eine braunrote Fläche, etwa so groß wie ein Markstück und fast ebenso vollkommen rund. Selbige war umgeben von einer schmalen, blaß-bräunlich gefärbten Schicht, in deren weiterer Umgebung das Mark weder in Farbe, noch in Konsistenz irgendwie vom Normalen abwich. Die Schnittfläche des Herdes hatte mit derjenigen einer Blutung Ähnlichkeit, fühlte sich jedoch beim Berühren trocken und fein-rauh an.

Das Präparat wurde mir von Prof. Dr. Oestreich zur genaueren Untersuchung übergeben.

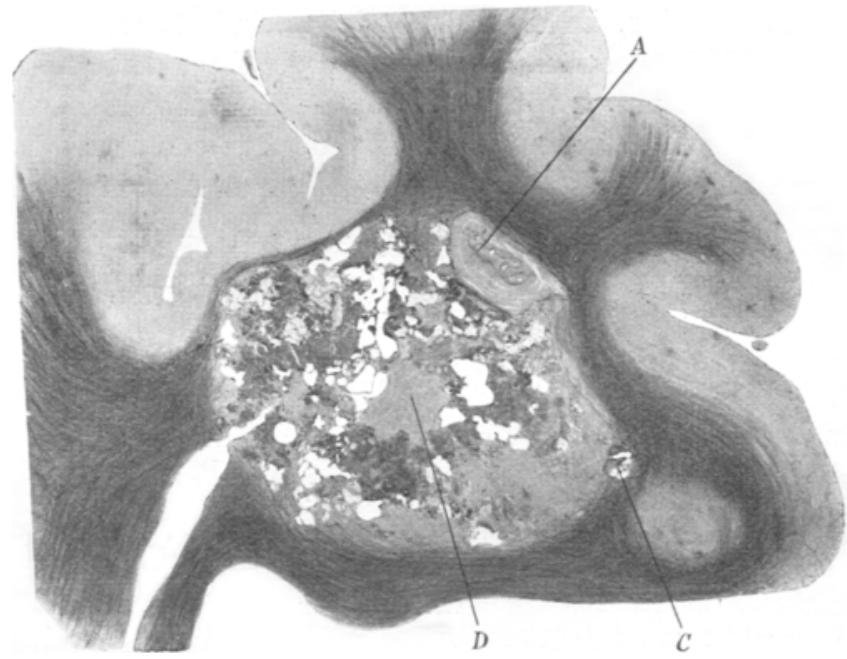

Fig. 1. Fünffache Vergrößerung. Pal-Weigert. A Geschlossenes Gefäß, welches sich später als divergierender Fortsatz entwickeln wird. D Balken. $C$ Offenes divergierendes Gefäß.

Die eine Hälfte wurde zum Zwecke einer mikroskopischen Untersuchung in situ mit einem großen Stück umgebender Hirnsubstanz herausgeschnitten und 24 Stunden in 10 proz. Formalin, dann 21 Tage in Müllersche Flüssigkeit gelegt, worauf Einbettung in Celloidin folgte.

Aus dem Block wurde eine Serie von $20 \mu$ dicken Schnitten hergestellt. Wegen der Härte und der leichten Zerreißbarkeit der Tumormasse war es unmöglich, Sohnitte dünner als $20 \mu$ anzufertigen. Abwechselnd wurden die Schnitte mit Pal-Weigert und mit Hämalaun-Eosin gefärbt. Hier und da wurde zwischendurch ein Schnitt mit Hämalaun-van Gieson gefärbt. Die Serie nimmt ihren Anfang mitten im Tumor und erstreckt sich nach dem Pol hin. Die Serie war vollständig. 
Da die Bedeutung des Tumors erst nach dem Schneiden offenkundig wurde, ist leider nur die eine Hälfte vollständig mikroskopisch verarbeitet worden, jedooh war die andere Hälfte makroskopisch ganz gleich beschaffen.

Aus der Gesamtheit der Serienschnitte ergibt sich, daß die Form der ganzen Geschwulst etwa länglich rundlich war.

Die Form ist derartig, daß sich nach allen Seiten hin Fortsätze erstrecken, am längsten bis $1 \mathrm{~cm}$ (cactusartig). Die Fortsätze verjüngen sich und laufen schließlich in ein stumpfes Ende aus.

Mikroskopisch ist die Geschwulst überall scharf gegen das Gehirngewebe abgegrenzt. Die der Geschwulst zunächst liegende Gehirnschicht besteht aus lockerem,

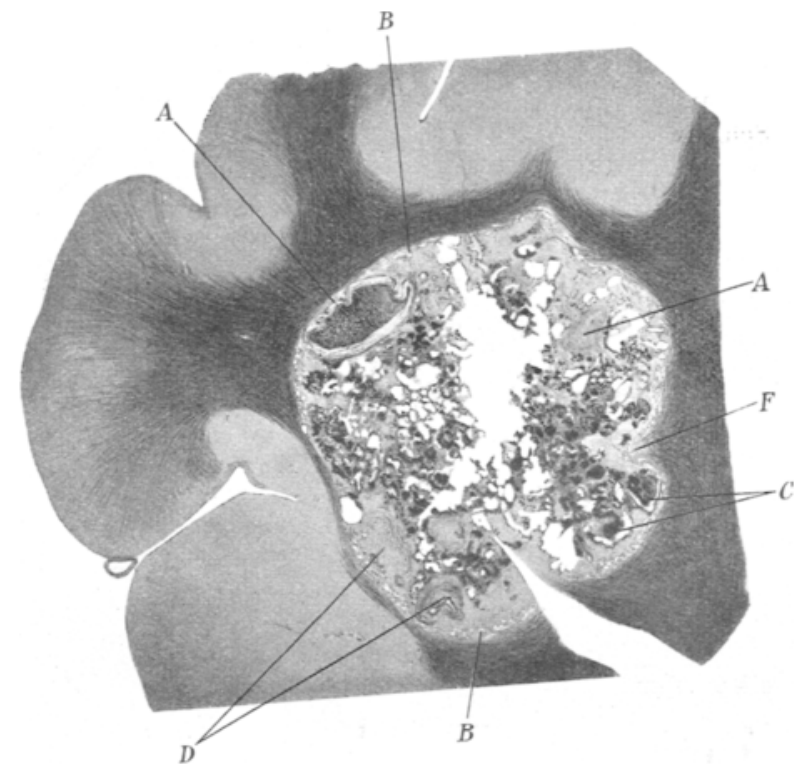

Fig. 2. Fünffache Vergrößerung. Pal-Weigert. A Geschlossenes Gefäß, welches sich später als Fortsatz entwickeln wird. $B$ Saum von Bindegewebe, $C$ Offenes divergierendes Gefa $B$, welches man bis zur kleinen Caplllare verfolgen kann. $D$ Anfang des in den Figg. 3 u. 4 zu sehenden Fortsatzes $D$. $F$ Eindringen von Nervenfasern an den Gliasaum heran.

kernreichem Gliagewebe, weiter nach außen iiegen viele sehr kleine Capillaren und viele, offenbar vermehrte Gliazellen; bis in diese Schicht hinein erstrecken sich auch die markhaltigen Nervenfasern.

Es besteht um diese Geschwulst herum nicht wie gewöhnlich bei anderen Geschwülsten eine Erweichungszone, sondern Geschwulst und Nervengewebe stoßen fast unvermittelt aneinander.

Die Geschwulst erweist sich als ein typisches Angioma cavernosum: balkiger Bau, Füllung der Räume mit Blut und Auskleidung der Wände mit Endothel. Die Balken bestehen aus echtem Bindegewebe, nicht aus Neuroglia. Innerhalb der Räume finden sich an einzelnen Stellen Thromben und Organisation (gleich wie in den Angiomen anderer Körperstellen). Die in die umgebende Gehirnsubstanz gehenden Fortsätze sind gefäßhaltig, bindegewebig, zapfenartig, auch verästelt. 
Sarkomatöse oder anders geartete Stellen haben sich nicht gefunden.

Die pathologische Histologie der kavernösen Blutgeschwülste verschiedener Gewebe lehrt, daß sie alle gleichartig sind; eine Bearbeitung eines Kavernoms der Leber, des Knochens, des Gehirns kann auch auf alle anderen Kavernome bezogen werden. Lusch ka erkannte diese Tatsache, als er ein Angioma cavernosum des Gehirns, welches dem unserigen ganz ähnlich lag und ebenso umschrieben war wie das unserige, beschrieb: er pfichtete damals Rokitans $\mathrm{k}$ y bei in der Behauptung, daß die kavernösen Blutgeschwülste als eigentliche Neubildungen anzusehen sind.

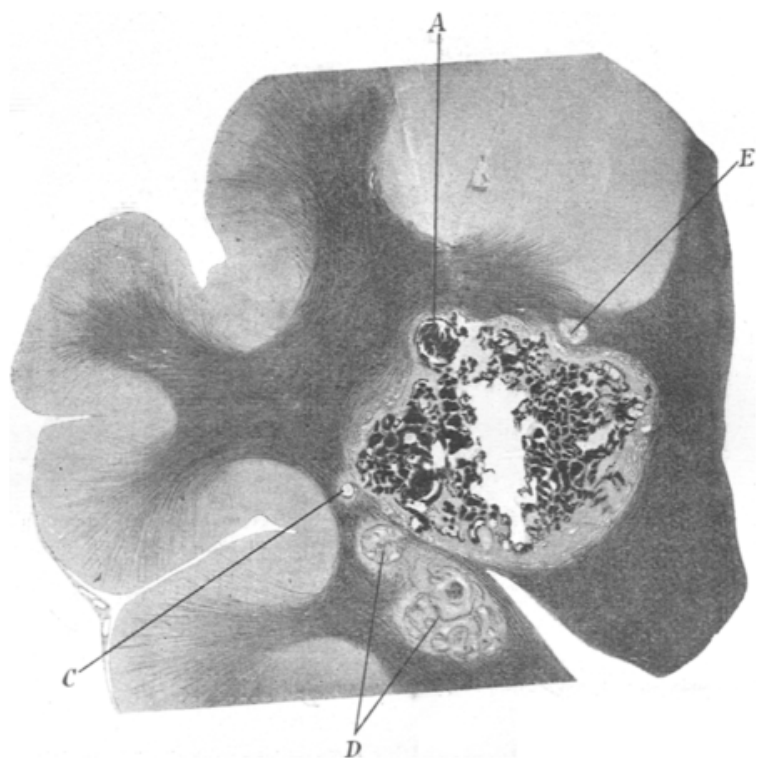

Fig. 3. Fünfache Vergrößerung. Pal-Weigert. A Thrombus im Maschenraum, welcher spáter als divergierender Fortsatz sich entwickeln wird. $C$ Offenes divergierendes GefäB. $D$ Fortsatz $D$, gesehen in den Figg. 2-4, welcher in seinem divergierenden Verlauf quer durchgeschnitten ist. $E$ Divergierender Fortsatz.

Virchow ${ }^{1}$ ) vertrat die Meinung, daß eine Wucherung des interstitiellen Bindegewebes in der Leber eine Atrophie der Leberzellen verursache und daß das ganze Gefäßsystem dieser Teile nach und nach eine kavernöse Ektasie bilde. Er beschrieb die Gefäßerweiterung mit Atrophie und Verdünnung der Gefäßwände, zugleich den Schwund des zwischenliegenden Gewebes, so daß zuletzt die Scheidewände an einzelnen Stellen zugrunde gehen, die verschiedenen Säcke zusammenfließen und eine unregelmäßige Höhlenbildung zustande kommt.

Eine Stauung in dem örtlichen Kreislauf wird als Ursache des An-

1) Ưber kavernöse (erektile) Geschwülste und Teleangiektasien. Virchows Archiv 6. 1854. 
gioma cavernosum angeführt. Scheffe $n^{1}$ ) behauptet, daß Stauung eine Erweiterung mit darauf folgenden Veränderungen in den Gefäßwänden verursacht und daß sich endlich eine Gruppe von Gefäßen, die unter sich verbunden sind, bildet.

Besonders bemerkenswert ist die Behauptung ${ }^{2}$ ), daß zuerst eine Blutung in den Geweben stattgefunden hat, und daß anstatt Resorption eine Organisation mit neugebildeten Blutgefäßen in der Form eines Angioms eintritt. Diese Gefäße vermehren sich, der Tumor wächst und somit wird durch Druck das ursprüngliche Gewebe zerstört.

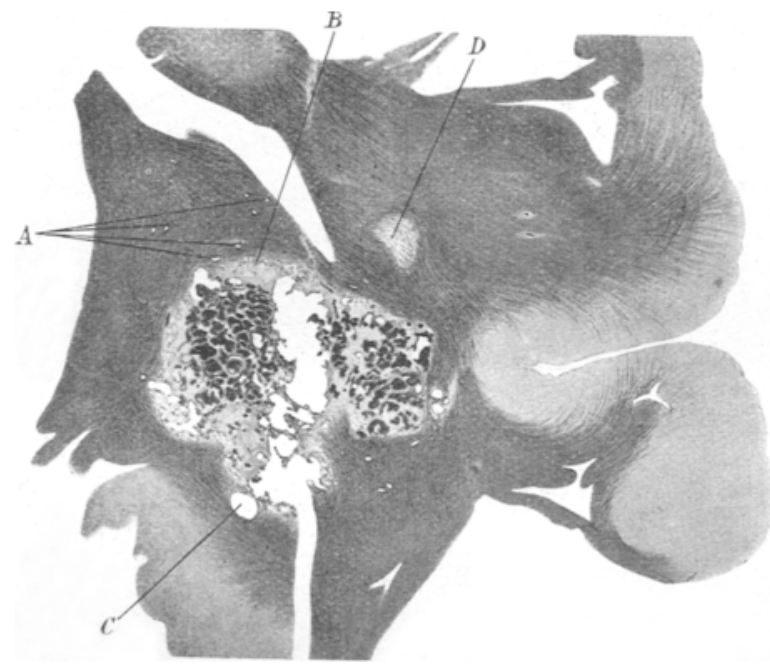

Fig. 4. Fünffache Vergrößerung. Pal-Weigert. A Kleine Gefäße mit Verkalkungen in den Wänden. $B$ Saum von Gliagewebe. $C$ Offene GefäBe, vom Tumor divergierend. $D$ Ende des in den Figg. 2 ı. 3 gesehenen Fortsatzes $D$.

Schmieden ${ }^{3}$ ) erklärte das Kavernom für embryonalen Ursprungs und für wachstumsunfähig. Er stützte sich auf die an einem Leberkavernom gemachten Beobachtungen.

Astwazat uroff ${ }^{4}$ ) hielt die Virchowsche Theorie über den Ursprung des Kavernoms für die zutreffende. Für ein Leberkavernom erschiene sie auch annehmbar, aber eine Wucherung von Bindegewebe innerhalb des Gehirns läßt sich nicht so leicht erklären. Astwazaturoff wurde zu seiner Ansicht bestimmt durch die Lage des von ihm beobachteten Tumors, welcher sich in der Hirnrinde dicht unterhalb der Oberfläche befand und mit der Pia unmittelbar verbunden war.

1) Beiträge zur Histogenese der Leberkavernome, Diss. Bonn 1897.

2) Felix Pilzer, Zur Genese des Angioma cavernosum. Archiv f. pathol. Anat. u. Physiol. 165. 1901.

3) Über den Bau und die Genese der Lebercavernome. Virchows Archiv 101, 373.

4) l. c.

z. f. d. g. Neur. u. Psych. o. XVI. 
Wo sich hingegen der Tumor in dem weißen Marklager des Centrum semiovale befindet und mit der Pia mater nicht in Verbindung steht, kann man sich eine Wucherung des Bindegewebes kaum vorstellen. [Fälle von Luschka ${ }^{1}$ ), Engelhart ${ }^{2}$ ), Bruns${ }^{3}$ ), und der meinge].

Stauung oder Blutung und eine ihnen folgende anormale Entwicklung von Blutgefäßen würden von Anfang an auffallende klinische Erscheinungen hervorrufen.

Aber alle Beschreibungen des Angioma cavernosum im Gehirn zeigen eine Krankengeschichte entweder ganz ohne Symptome oder mit einem

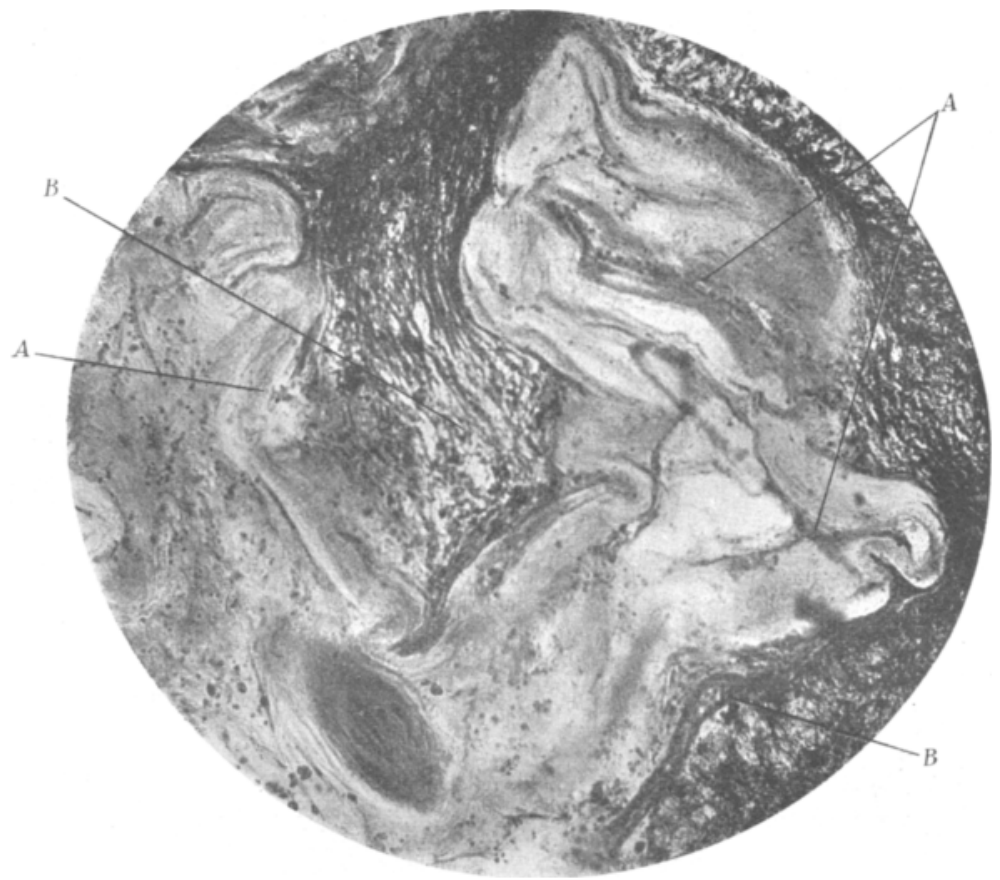

Fig. 5. Alaunhämatoxylin-Fosin. A Konvolutionen der Bindegewebswände des in den Figuren 2, 3, 4 gesehenen divergierenden Fortsatzes $D, B$ Saum von Gliagewebe.

Symptomkomplex langsam und allmählich im Entstehen, und in dem weiteren Verlauf mit Intermissionen ${ }^{4}$ ).

Weniger durch besondere neue an unserem Präparat gewonnene Beobachtungen beeinflußt (denn es bot in dieser Beziehung nichts Uber-

1) l. c.

2) $1 . \mathrm{c}$

3) $1 . \mathrm{c}$.

4) Oppenheim, Über klinische Eigentümlichkeiten kongenitaler Hirngeschwülste. Neurol. Centralbl. Nr. 1, S. 7. 1913. 
zeugendes dar), als nach einer gründlichen Durchsicht der Literatur (mit Ausschaltung aller weniger wahrseheinlichen Theorien) möchten wir uns der Mehrzahl derjenigen, die Angiome beschrieben haben, anschließen in der Meinung, daß eine embryonale Mißbildung eines speziellen Teiles des Vasomotorensystems auftritt, daß infolgedessen eine anormale Vermehrung der Gefäße entsteht und sich so ein Angioma cavernosum bildet. Die bereits im embryonalèn Leben angelegte Störung entwickelt sich entweder sehr langsam oder sie bleibt überhaupt zunächst lange Zeit stationär, um dann mit einem Male zu wachsen. Sekundäre Veränderungen, d. h. Thrombose, Verkalkung, Zerreißung der Gefäßwände können auftreten und sind oft der Grund einer mehr oder weniger intensiven Verschlimmerung klinischer Symptome.

Bezüglich der Entstehung und der Entwicklung der kavernösen Geschwülste möchten wir noch Ribbert' ${ }^{1}$ ) anführen, seine Beobachtungen beziehen sich auf ein Kavernom der Leber:

„Ich bin der Meinung, daß Gefäß-und Bindegewebsneubild ung von vornherein parallel gehen. Das Wachstum der Kavernome $\mathrm{muß}$ sich dann allerdings in der Hauptsache so vollziehen, wie es Virchow und Rindfleisch angeben. Denn da die Bindesubstanz die äußersten Teile der Neubildung einnimmt, so muß sie es auch sein, die zunächst in das angrenzende Lebergewebe vordringt, aber freilich im gleichen Schritte gefolgt von den mitwachsenden Bluträumen, die also meiner Meinung nach nicht erst sekundär im Bindegewebe entstehen. Diese Vergrößerung der Kavernome geht nun im Laufe so allmählich vor sich, daß deutliche Verdrängungserscheinungen am anstoßenden Lebergewebe nicht nachweisbar sein können."

Die mikroskopische Untersuchung unserer Präparate zeigt deutlich, daß sich zahlreiche Bindegewebsfibrillen von dem Rande des Tumors aus in das umgebende Gewebe hinein erstrecken, diese treffen sich und zwischen ihnen liegen dann Inseln von Gliagewebe, welche somit vollständig eingeschlossen sind. (Virchow machte dieselbe Beobachtung bei einem Leberkavernom.) Wo die Fibrillen genügend entwickelt'sind, sieht man kleine Capillaren; mit der Zunahme der Bindegewebsfibrillen erfolgt eine Vermehrung und Vergrößerung der Blutgefäße, so daß dicht an dem Tumorrande entlang kleine Stellen von Glia teilweise von bluterfüllten Räumen umgeben sind; innerhalb des Tumorrandes, nicht weit davon entfernt, sieht man Inseln von Gliagewebe, welche ganz von dem bluterfüllten Maschenwerk umgeben sind.

Der Eindruck, den die älteren Autoren Luschka, Rokitansky und andere gewonnen hatten, daß das Angioma cavernosum in seiner Entstehung ein umschriebener, von dem allgemeinen Vasomotoren-

1) U̇ber Bau, Wachstum und Genese der Angiome usw. Ar chiv f. pathol. Anat. u. Physiol. 151, 381. 
system unabhängiger Tumor sei, war zweifellos einer fehlerhaften Technik zuzuschreiben, denn es ist durch Serienschnitte und genaue Prüfung histologischer Präparate festzustellen, daß der Tumor mit dem Vasomotorensystem in Verbindung steht, sich aber insofern von anderen Arten der Blutgeschwülste unterscheidet, daß er nicht mit einem einzigen großen Gefä $\beta$, sondern mit vielen kleineren direkt verbunden ist. Diese Art der Kommunikation ist in unseren Präparaten deutlich zu sehen.

Nach dieser Richtung hin haben wir sogar eine Beobachtung gemacht, welche bisher erst ein mal von einem Hirn-Kavernom erwähnt worden ist: Luschka beschreibt Ausläufer, welche sich von den Seiten des Tumors in das Hirngewebe hinein erstrecken; er bezeichnet sie als Hohlkölbchen und schreibt ihnen das weitere Wachsen des Tumors zu. Bei der Betrachtung unserer Präparate mit der Lupe fallen gewisse Stellen auf, welche sich als Fortsätze von Bindegewebe darstellen und sich von dem Tumor aus nach allen Richtungen erstrecken. Wenn diese Fortsätze stärkerer Vergrößerung unterworfen werden, bilden sie eine Fortsetzung nicht der begrenzenden Wand des Tumors, sondern bald eines Balkens, bald eines Maschenraumes. Das Aussehen des einen oder anderen blutgefüllten Maschenraumes ändert sich, die Wände erscheinen dicker, das, was zuerst eine Masse unterscheidbarer Blutzellen zu sein schien, wird homogen und zeigt beginnende Organisation. Das Ganze nähert sich allmählich der Peripherie des Tumors und geht schließlich in die Hirnsubstanz über, wo es sich immer weiter fortsetzt unter beständiger Entfernung von dem Tumor, bis es schließlich in ein stumpfes Ende ausläuft.

Diese Fortsätze sind von ganz verschiedener Länge. An allen Fortsätzen erkennt man, daß sich ein Netzwerk von Bindegewebefibrillen aus der umgebenden Wand in die zentrale Masse hineindrängt und die Wand selber allmählich verschwindet, bis schließlich nur noch eine Säule von Bindegewebefibrillen als loses Netzwerk besteht, in deren unmittelbarer Umgebung mannigfache kleine Capillaren liegen. An einer Stelle sieht man noch den letzten Rest eines derartigen Fortsatzes, umgeben von einem vollkommenen Ring, dieser besteht aus winzigen Capillaren, welche miteinander parallel und zu der Achse des Fortsatzes rechtwinklig verlaufen.

Alle Fortsätze werden von der schmalen Gliawand, welche in dem Haupttumor beobachtet wurde, umgeben, und die Nervenfasern erstrecken sich bis an diese schmale Wand heran.

Eine weitere Untersuchung der Serie lehrt, daß die Gefäße, welche mit dem Tumor in Verbindung stehen, dieselbe Richtung verfolgen, wie die Fortsätze. Wir sehen Blutgefäße aus sehr kleinen Muttergefäßen hervorgehen, nach dem Tumor hin verlaufen und, dort angelangt, hinein- 
treten, entweder als selbständige Maschen oder in Verbindung mit schon bestehenden Maschen.

Sehr auffallend ist es bei diesen Gefäßen, daß das Lumen auf dem Wege von dem Muttergefäß zu dem Tumor stets an Größe zunimmt, so daß z. B. am Tumor das Lumen fünfmal größer ist, als das Lumen des Gefäßes, dem es entsprang. Man darf bei einem derartig erweiterten Flußbett annehmen, daß die Beförderung einen kolossalen Kraftaufwand seitens des Muttergefäßes erfordern muß und daß der Blutstrom sehr empfindlich ist gegen die geringsten Schwankungen des vasomotorischen Tonus, ferner daß leicht Blutstillstand und Gerinnung des Blutes mit darauf folgender Organisation eintreten könnte.

Die divergierenden Fortsätze dürfen nicht als Auswüchse in dem Sinne von Neubildungen betrachtet werden, sondern eher als eine Rückbildung schon bestehender Teile.

Bei einem Angioma cavernosum des Gehirns sind zweierlei Vorgänge zu unterscheiden - ein progressiver und ein regressiver. Der progressive Vorgang äußert sich in dem Herauswachsen von Bindegewebefibrillen aus den Tumorwänden und schreitet langsam vorwärts, während der regressive Vorgang in der V'erschließung der Gefäße besteht und sich verhältnismäßig schnell abspielt, wodurch sich vielleicht die spontane Remission der klinischen Symptome, die schon verschiedentlich erwähnt worden ist, erklären läßt.

Unser Tumor zeigte keine Verbindung mit der Oberfläche des Gehirns oder mit Gefäßen, die mit äußeren Kopfteilen zusammenhängen.

Vom chirurgischen Gesichtspunkte aus ist der Fall insofern von großer Bedeutung, daß, wie schon Kra u se ${ }^{1}$ ) betont hat, durch die Ausführung einer Hirnpunktion, um Material zur Diagnose zu erhalten, eine Blutung herbeigeführt werden kann, und daß gegen die operative Entfernung des Tumors kein Einwand erhoben werden kann.

\section{Literaturverzeichnis.}

1. Rossolino, Zum Ausgang von Gehirnoperationen. Archiv f. Psych. 29, 528.

2. Engelhart, Zur Frage der Dauerheilung nach operativer Behandlung der traumatischen Jacksonschen Epilepsie. Deutsche med. Wochenschr. Nr. 3, S. 7.1904 .

3. Creite, Zur Pathogenese der Epilepsie (multiple Angiome des Gehirns mit Ossification). Münch. med. Wochenschr. 1903.

4. Blank, Ein Fall von Rankenangiom des Gehirns. Münch. med. Wochenschr. 1910, Nr. 9, S. 465.

5. Kra use, Fedor, Chirurgie des Gehirns und Rückenmarks 1, 55, 154. 1908.

6. Inojensky u. Myslwecek, Angioma arteriale racemosum arteriae cerebelli inferior, anterior sinistrae et angioma racemosum arteriae cerebri mediae sinistrae. Revue v. neurologii H. 5 u. 8.

7. Pean, Bull. Acad. méd. 25, 881-883, T. 3. 1891.

1) Chirurgie des Gehirns und Rückenmarks 1, 154. 1908. 
8. Pilzer, Zur Genese des Angioma cavernosum. Archiv f. pathol. Anat. u. Physiol. 165, 427.

9. Kalischer, Berliner klin. Wochenschr. 1897, S. 1059.

10. Luschka, Kavernöse Blutgeschwülste des Gehirns. Archiv f. pathol. Anat. u. Physiol. 6, 458.

11. Virchow, Utber kavernöse Geschwülste und Teleangiektasien. Archivf. pathol. Anat. u. Physiol. 6, 525.

12. Ribbert, U̇ber Bau, Wachstum und Genese der Angiome usw. Archiv f. pathol. Anat. u. Physiol. 151, 381.

13. Emanuel, Deutsche Zeitschr. f. Nervenheilk. 14, 288.

14. Deetz, U̇ber ein Angioma arteriale racemosum im Bereich der Arteria corporis callosi. Archiv f. pathol. Anat. u. Physiol. 168, 341. 1902.

15. Str u p pler, Über das kavernöse Angiom des Großhirnes. Münch. med. Wochenschrift 1900, Nr. 37, S. 1269.

16. Bielschowsky, Zur Histologie und Pathologie der Gehirngeschwülste. Deutsche Zeitschr. f. Nervenheilk. 22.

17. Bruns, Die Geschwülste des Nervensystems. S. 30. Berlin 1908.

18. Astwazaturoff, UUber die kavernöse Blutgeschwulst des Gehirns. (Zur Kasuistik der Pseudomeningitis.) Frankfurter Zeitschr. f. Pathol. 4, 481. H. III.

19. Hadlich, Ein Fall von Tumor cavernosus des Rückenmarkes. Virchows Archiv 172.

20. Lorenz, Kavernöses Angiom des Rückenmarkes mit tödlicher Blutung. Inaug. Diss. Jena 1901/02.

21. Beneke, R., Kasuistische Beiträge zur Geschwulstlehre. Archiv f. pathol. Anat. u. Physiol. 119.

22. O p pen hei m, Ủber klinische Eigentümlich keiten kongenitaler Hirngesch wülste, Neurol. Centralbl. 1913, Nr. 1, S. 7.

23. - Monographie der Hirngeschwülste II. Aufl., 1902, S. 222.

24. Scheffen, Beiträge zur Histogenese der Leberkavernome. Diss. Bonn 1897.

25. Sehmieden, Utber den Bau und die Genese der Leberkavernome. Virchows Archiv 101, 373.

26. Rokitansky, Lehrbuch der pathologischen Anatomie 2. 1856.

27. Rindfleisch, Lehrbuch der pathologischen Gewebe-Lehre S. 190. 